Research Paper

\title{
Successful Two-Stage Exchange Arthroplasty for Periprosthetic Infection Following Total Knee Arthroplasty: The Impact of Timing on Eradication of Infection
}

Ines Vielgut, MD, PhD ${ }^{1 凶}$, Gerold Schwantzer, $\mathrm{Mag}^{2}$, Andreas Leithner, Prof, MD ${ }^{1}$, Patrick Sadoghi, Prof, MD, PhD ${ }^{1}$, Uldis Berzins, MD1, Mathias Glehr, Prof, MD ${ }^{1}$

1. Department of Orthopaedics and Trauma, Medical University of Graz, Graz, Austria

2. Institute for Medical Informatics, Statistics and Documentation, Medical University of Graz, Graz, Austria

$\triangle$ Corresponding author: Ines Vielgut, MD, PhD. Department of Orthopaedics and Trauma, Medical University of Graz, Auenbruggerplatz 5, 8036 Graz, Austria. Email: ines.vielgut@gmx.at; Phone: +4331638581946

(C) The author(s). This is an open access article distributed under the terms of the Creative Commons Attribution License (https://creativecommons.org/licenses/by/4.0/). See http://ivyspring.com/terms for full terms and conditions.

Received: 2020.05.01; Accepted: 2020.11.30; Published: 2021.01.01

\begin{abstract}
Background: Periprosthetic joint infection (PJI) represents a serious complication following total knee arthroplasty. In the setting of chronic infections, the two-staged approach has traditionally been the preferred treatment method. The aim of this study was to determine the optimal period of rest between the first and second stage. Furthermore, we analyzed potentially outcome-relevant parameters, such as general and local conditions and the presence of difficult-to-treat or unidentified microorganisms, with regard to their impact on successful treatment of PJI.

Patients and Methods: We performed a retrospective analysis of prospectively collected data for all patients treated for PJI at our institution. Seventy-seven patients who had undergone two-stage revision arthroplasty for PJI of the knee were included into the study. Antibiotic-loaded cement spacers were used for all patients.

Results: After a median follow-up time of 24.5 months, infection had reoccurred in 14 (18.7\%) patients. A prolonged spacer-retention period of more than 83 days was related to a significantly higher proportion of reinfections. Furthermore, significant compromising local conditions of the prosthetic tissue and surrounding skin, as well as repeated spacer-exchanges between first- and second-stage surgery, negatively influenced the outcome. Neither the patients' age nor gender exerted a significant influence on the outcome regarding reinfection rates for patients' age or gender.

Conclusions: We observed the best outcome regarding infection control in patients who had undergone second-stage surgery within 12 weeks after first-stage surgery. Nearly $90 \%$ of these patients stayed free from infection until the final follow-up. An increased number of performed spacer-exchanges and a bad local extremity grade also had a negative impact on the outcome.
\end{abstract}

Key words: Total knee arthroplasty; Prosthetic knee joint infection; Antibiotic-augmented joint spacer; Two-stage revision procedure

\section{Introduction}

Despite continuous innovations and improvements in knee arthroplasty, the occurrence of prosthetic joint infections (PJI) has not decreased. Although the overall PJI incidence following total knee arthroplasty is low (1-2\%), PJI represents a serious complication with potentially devastating effects to the patients [1-3]. Two-stage exchange arthroplasty has been the procedure of choice for chronic infections. Although this procedure has consistently demonstrated infection control rates of 
$80-90 \%$, there are still cases where this method fails [3]. There are several possible explanations. First, there are marked differences regarding individual circumstances (resistant or difficult to treat microorganisms, worse local and general conditions). Second, the treatment protocol for two-stage exchange arthroplasty may vary depending on geographic and/or empiric experiences of the treating department [2-7]. For example, to our knowledge there are no specific recommendations concerning the length of joint-spacer retention [1, 3, 8-9]. We hypothesized that the duration of spacer-retention plays an essential role for treatment success.

In order to determine the optimal period of rest between the first and second stage, we analyzed the data of 77 consecutive patients who had undergone two-stage exchange arthroplasty for prosthetic knee joint infections with regard to successful infection control. Furthermore, we analyzed potentially outcome-relevant parameters (general and local conditions, difficult-to-treat microorganisms, and age) with regard to their impact on successful treatment of PJI.

\section{Patients and Methods}

We included a consecutive series of 77 patients who had undergone two-stage revision arthroplasty for PJI in the study. The series comprised 35 men and 42 women with a mean age of 64.9 years (range, 31.3-82.4 years). The right knee was affected in 37 cases, the left knee in 40 cases. The median observation period was 24.5 months (range, 6-107 months).

\section{Diagnosis}

Currently, the Musculoskeletal Infection Society (MSIS) criteria is the gold standard for infection diagnosis [10, 11]. We assessed the patients' history and clinical findings with regard to previous interventions, symptom duration, as well as general and local comorbidities. We obtained inflammation indices (complete blood count with total and differential leukocyte count, C-reactive protein, fibrinogen, and interleukin-6 [IL-6]), as well as blood cultures in cases of fever or other systemic signs of infection [12]. We sent synovial fluid, obtained from joint aspiration, for synovial fluid white blood cell (WBC) count and bacterial cultures. We took standardized radiographs (complete leg, knee in two planes, patella tangential and compared them to previous radiographs, if available, to assess signs of loosening.

Even if the causing bacteria could not be identified preoperatively, patient's history and clinical findings, as well as the presence of elevated inflammation markers and synovial WBC, were sufficient tools in order to obtain security about whether infection was present or not. In cases of inconclusive clinical, laboratory, or bacteriological findings (low-grade infections), we also performed a $99 \mathrm{mTc}$-leukocyte scintigraphy. We diagnosed PJI according to the MSIS criteria. Furthermore, we documented the general health and local wound condition (may contribute to planning intraoperative graft-placement to ensure optimal conditions in the upcoming techniques for PCL reconstruction.

\section{Surgical technique}

\section{First-stage procedure}

First-stage procedure included the collection of prosthetic specimens (synovia, synovial fluid, and joint capsule) for cultural examination, followed by a thorough debridement and removal of all implants, which we sent for sonication. Subsequently, we placed an antibiotic-loaded bone cement (polymethylmethacrylate [PMMA]) as a static spacer. We used 80-120 grams of PALACOS cement (Heraeus, Germany), which was loaded with 1 gram of vancomycin per $40 \mathrm{~g}$ of Palacos in most of the cases (94.8\%). In some cases, we used different antibiotics, according to the pathogen-specific antibiogram: Antibiotic loading of the spacer comprised vancomycin in almost all cases Tazobactam was applied in $2.6 \%$ of cases, while vancomycin/refobacin or teicoplanin and cefuroxime was each used in 1.3\% of cases.

\section{Spacer retention period}

A period of rest between first- and second-stage surgery has been considered necessary in order to ensure treatment success due to infection eradication $[13,14]$. However, the optimal spacer retention period remains unclear, although several reports have suggested an interval of 6 weeks. In our series, first-stage surgery was followed by an interval of systemic antibiotic administration (usually 6-8 weeks, as recommended in the literature) until the infection was clinically eradicated before the second procedure was performed $[3,12,13,14]$.

\section{Second-stage procedure}

Infection was considered to be eradicated with the resolution of clinical signs and symptoms, as well as improvement of laboratory infection values. According to our experience, although defining cut-off values has seemed to be challenging in the recent past, a linear decrease followed by steadiness of all infection values within the high-normal to slightly increased range appears to be indicative for 
treatment success [15].

If those criteria were met, we executed second-stage surgery. We again performed a thorough soft tissue debridement and synovectomy. We removed the joint spacer with care in order to preserve as much bone stock as possible. Finally, after careful debridement of the posterior joint, we performed revision arthroplasty (semi-constrained, stemmed components) in all of the cases in order to achieve stability.

\section{Postoperative care}

The primary goal of the follow-up care was the early detection of re-infection. These follow-up assessments comprised physical examinations, regular laboratory tests (complete blood count, C-reactive protein, and fibrinogen), and imaging procedures, including plain radiographs of the knee in two planes after 6 weeks, 3, 6 and 12 months, and annually thereafter. All patients were followed up every six months for at least two years after second stage surgery.

\section{Statistical analysis}

We entered data into a computerized database and analyzed it. Continuous variables are reported as the median with interquartile range (IQR) or range (minimum-maximum), except for the age, which is described as the mean with standard deviation (SD). Categorical data is displayed as frequencies with percentages in parentheses.

To separate patients with an optimum spacer retention period from patients with a prolonged spacer retention period we used the "maximally selected rank statistic" method [16], calculated with the $\mathrm{R}$ package "maxstat" [17]. This method locates the cut-point that maximizes a logrank test statistic as usually applied in connection with a time-to-event analysis. The effect of the spacer retention period on time to reinfection was therefore assessed with a Kaplan-Meier plot with Mantel-Cox logrank test and the hazard ratio for patients with a prolonged spacer retention period of getting a reinfection compared to patients with optimum spacer retention period was assessed with a Cox proportional hazard model adjusted for age and sex.

To determine the statistical significance of group differences, we used the Wilcoxon rank-sum test for metric parameters. The relationship between categorical parameters is presented in contingency tables and analyzed with Pearson's chi-squared test. In either case, we determined exact $p$ values; we considered $p \leq 0.05$ to be statistically significant. Computations were performed using the statistical package IBM SPSS Statistics for Windows (Version 24.0, International Business Machines Corporation, Armonk, NY, USA) and R, version 3.3.0.

\section{Results}

\section{Pre-operative diagnostics and assessment}

Clinical manifestations ranged from reduced mobility, impaired range of motion, pain, swelling, and/or redness of the affected region to systemic signs of inflammation or even sepsis. The main clinical symptoms were pain, joint swelling and effusion, overheating, and movement restriction of the affected joint. The median time period between primary joint arthroplasty and onset of the symptoms was 23.6 months (range, 6 months to 336 months).

\section{Pre-operative laboratory inflammation parameters}

The median preoperative C-reactive protein concentration was $61.1 \mathrm{mg} / \mathrm{L}$, the median leukocyte count was 7920 cells $/ \mu \mathrm{L}$, the median fibrinogen concentration was $589 \mathrm{mg} / \mathrm{dL}$, and the median IL-6 concentration was $31.5 \mathrm{pg} / \mathrm{dL}$.

We performed pre-operative joint-aspirations in all patients. An organism could be detected either for pre-operative or both pre- and intra-operative samples in 54 cases $(70.1 \%)$. In 13 patients $(16.9 \%)$, bacteria determination was exclusively by implant sonication. The most common identified organisms were staphylococci, followed by streptococci, gram negative rods, and mixed cultures of organisms (Table 1). In $23(29.8 \%)$ of the patients, the infection-causing germ was unidentified.

Table 1: Number of patients with determined infection-causing microorganisms

\begin{tabular}{lll}
\hline Infection-causing microorganisms & $\mathrm{n}$ & $\%$ \\
E. faecalis & 3 & 3.9 \\
E. faecium & 1 & 1.3 \\
E. faecium, E. faecalis & 1 & 1.3 \\
Group C streptococci & 1 & 1.3 \\
Group G streptococci & 1 & 1.3 \\
MRSA & 1 & 1.3 \\
P. aeruginosa & 2 & 2.6 \\
P. aeruginosa, Bacillus cereus & 1 & 1.3 \\
Pseudomonas aeruginosa, Finegoldia magna & 1 & 1.3 \\
S. aureus & 13 & 16.9 \\
S. aureus, E. faecalis & 1 & 1.3 \\
S. capitis & 3 & 3.9 \\
S. epidermidis & 8 & 10.4 \\
S. hominis & 1 & 1.3 \\
S. hominis, E. faecalis & 1 & 1.3 \\
S. hominis, S. haemolyticus & 1 & 1.3 \\
S. lugdunensis & 1 & 1.3 \\
Microorganisms detected by sonication (not detected in tissue & $\mathrm{n}$ & $\%$ \\
samples/joint aspirations) & \\
P. acnes & 1 & 1.3 \\
P. aeruginosa & 1 & 1.3 \\
S. aureus & 4 & 5.2 \\
S. capitis & 2 & 2.6 \\
S. epidermidis & 5 & 6.5 \\
Undetected microorganisms & 2329.8 \\
\hline The microorganisms were detected either via tissue samples/joint aspiration on the \\
method of sonication. The table shows the frequency (n) and relative proportion of \\
the total patient number (\%). & \\
&
\end{tabular}




\section{Spacer-retention and Second-Stage Management}

After first-stage surgery, we continuously evaluated all patients in terms of clinical and laboratory improvements. The median overall spacer retention-period was 10.6 weeks (IQR 8.0 weeks). One additional spacer exchange was necessary in 15 patients (19.7\%). Two exchanges were performed in two patients $(2.6 \%)$ due to persisting signs and symptoms of infection. All patients underwent second-stage surgery.

\section{Outcome}

\section{Rate of reinfection and infection-related complications}

We observed an $18.7 \%(\mathrm{n}=14)$ rate of reinfection until a median time of 19.5 months (IQR 38.9; range, 0-63.9 months). In 11 of these cases, we performed two-stage revision. Two patients underwent transfemoral amputation due to worse local and general conditions. One patient died before completion of the two-stage protocol. Most patients $(n=61,81.3 \%)$ stayed infection free until a median follow-up time of 24.6 months (IQR 58.8; range, 6-106.9 months).

\section{Timing of reimplantation}

The maximally selected rank statistic method yields a maximum cut-point at 83 days, indicating an optimum spacer retention period of less than 12 weeks. Thirty-nine patients had an optimal and 35 patients a prolonged (more than 12 weeks) spacer retention period. Time to event analysis shows that patients with an optimum spacer retention period have a higher probability of staying free from reinfections than patients with a prolonged spacer retention period $(p=.005)$ (Figure 1). In a proportional hazard modell adjusted for age and sex (Cox regression) we found a hazard ratio of 6.1 (95\% CI 1.6 - 22.9) indicating a 6-fold higher risk of getting a reinfection for patients with a prolonged spacer retention period compared to patients with an optimal spacer retention period $(p=.007)$. Patients with a prolonged spacer retention period showed a higher proportion of reinfection (31.4\%) compared to patients with an optimal spacer retention period $(7.7 \%)(\mathrm{p}=0.016)$.

\section{Impact of individual parameters}

Significant compromising local conditions of the periprosthetic tissue and surrounding skin negatively influenced the outcome. Patients classified as McPherson [10] local extremity grade 3 showed statistically significant more reinfections following two-stage revision surgery for PJI of the knee $(50 \%)$ as patients with McPherson grade $2(16.3 \%)$ or grade 1 who showed no infections at all $(\mathrm{p}=0.005)$ (Table 2).

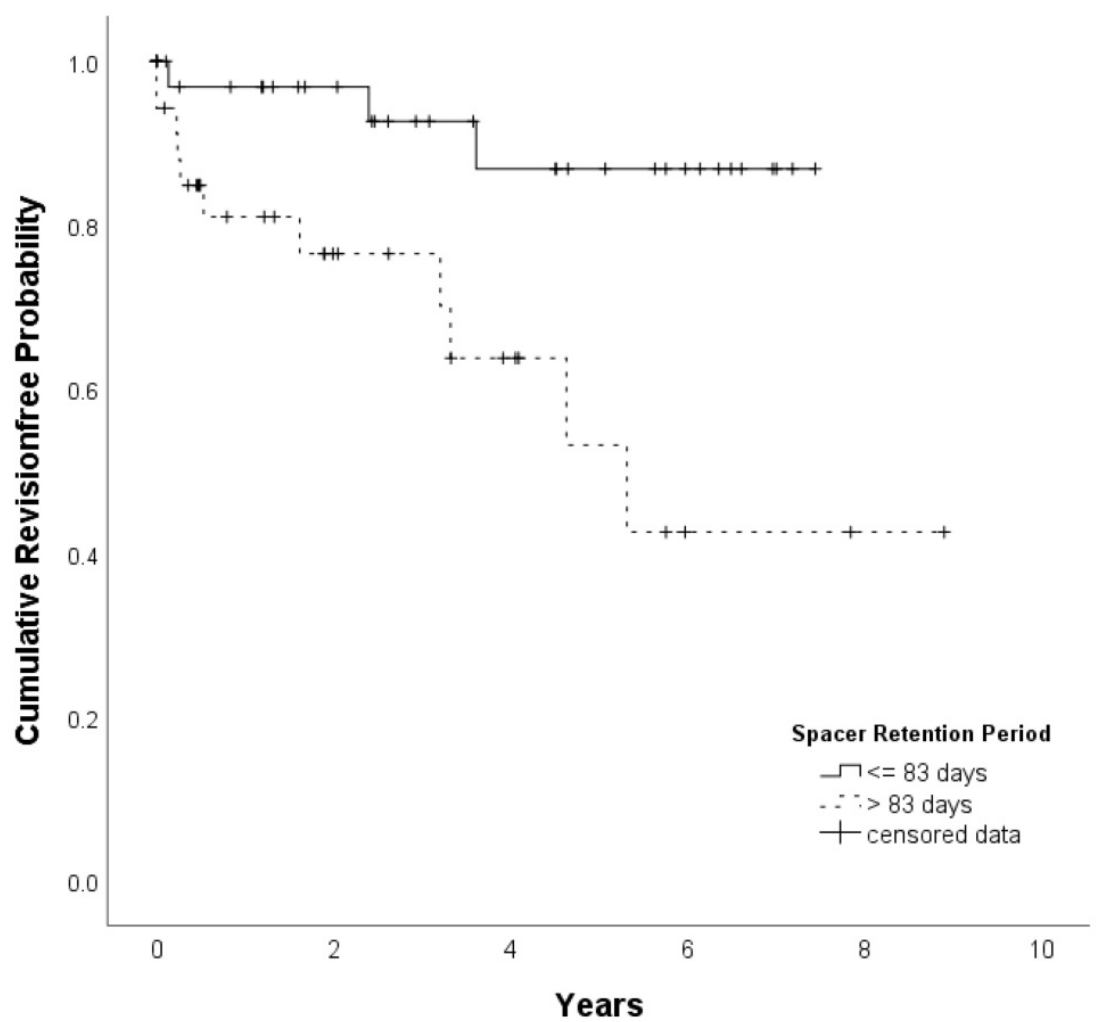

Figure 1: Kaplan-Meier plot of patients with an optimal (less than 83 days) and prolonged (more than 83 days) spacer retention period. The assessed optimal spacer retention period of less than 83 days showed significant better results compared to the group with prolonged intervals $(p=.005)$. $86.9 \%$ of all patients with an optimal retention period stayed free from infection by 5 years of follow-up time (vs. $53.1 \%$ for patients with prolonged spacer retention periods) 
Table 2: Reinfection rates following two-stage revision in relation to the local extremity grade

\begin{tabular}{lllllll}
\hline & \multicolumn{2}{l}{ Reinfection } & \multicolumn{2}{l}{ no Reinfection } & \multicolumn{2}{l}{ Total } \\
\hline Local extremity grade & $\mathrm{n}$ & $\%$ & $\mathrm{n}$ & $\%$ & $\mathrm{n}$ & $\%$ \\
\hline $\mathbf{1}$ (uncompromised) & 0 & $0.0 \%$ & 14 & $100.0 \%$ & 14 & $100 \%$ \\
$\mathbf{2}$ (compromised) & 8 & $16.3 \%$ & 41 & $83.7 \%$ & 49 & $100 \%$ \\
$\begin{array}{l}\mathbf{3} \text { (significant } \\
\text { compromised) }\end{array}$ & 6 & $50.0 \%$ & 6 & $50.0 \%$ & 12 & $100 \%$ \\
Total & 14 & $18.7 \%$ & 61 & $81.3 \%$ & 75 & $100 \%$ \\
\hline
\end{tabular}

Patients classified as grade 3, according to McPherson et al., showed more reinfections $(50 \%)$ than grade $2(16.3 \%)$ or grade 1 patients $(0 \%)$. This was statistically significant $(\mathrm{p}=0.005)$.

$\mathrm{N}$ defines the number of patients and \% the relative proportion of patients for each local extremity grade.

A further negative predictor was the number of performed spacer-exchanges between first- and second-stage procedure. An increasing number of spacer exchanges (once, twice, 3 times) was significantly associated with a higher rate of reinfection $(10.5 \%, 40.0 \%$ and $100 \%$, respectively) ( $\mathrm{p}=$ 0.001; Table 3). Neither the patients' age nor gender significantly influenced the outcome regarding reinfection based on the preoperative inflammatory markers.

Table 3: Reinfection rates following two-stage revision in relation to the number of performed spacer exchanges

\begin{tabular}{lllllllll}
\hline \multicolumn{7}{c}{ Number of performed spacer exchanges } \\
\hline & $\mathbf{1}$ & \multicolumn{2}{l}{$\mathbf{2}$} & & $\mathbf{3}$ & \multicolumn{3}{l}{ Total } \\
\hline & $\mathrm{n}$ & $\%$ & $\mathrm{n}$ & $\%$ & $\mathrm{n}$ & $\%$ & $\mathrm{n}$ & $\%$ \\
\hline $\begin{array}{l}\text { Reinfection } \\
\text { No }\end{array}$ & 6 & $10.5 \%$ & 6 & $40.0 \%$ & 2 & $100.0 \%$ & 14 & $18.9 \%$ \\
$\begin{array}{l}\text { Reinfection } \\
\text { Total }\end{array}$ & 51 & $89.5 \%$ & 9 & $60.0 \%$ & 0 & $0.0 \%$ & 60 & $81.1 \%$ \\
\hline
\end{tabular}

$\mathrm{N}$ describes the number of patients and \% the relative proportion of patients who have undergone the respective number of performed spacer exchanges.

\section{Discussion}

Although still controversial in the relevant literature, two-stage revision arthroplasty remains the most applied procedure for chronic periprosthetic infections. Antibiotic-loaded cement spacers have become an established method for infection control. The use of high-dose antibiotic-loaded cement as a temporary joint spacer seems to increase the potential for local infection eradication during the time between implant removal and before the second-stage surgery, which aims to restore function and mobility by the use of knee arthroplasty implants once again $[3,18$, 19]. While two-stage revision is still considered to be the gold standard in the treatment of PJI, especially in the United States, there is growing popularity for the one-stage approach $[13,20,21]$. This phenomenon might be due to the fact that one-stage revision, compared to two-stage revision, has the potential to reduce the overall surgical burden on patients as well as healthcare costs $[22,23]$. Despite these obvious advantages of single-stage revision surgery for PJI, this procedure seems to only be suitable for healthier patients with better bone stock and less virulent organisms only [13].

The goal of surgery must be the complete removal of all infected tissue and implants to eliminate the biofilm and ensure the efficacy of the postoperative antibiotic therapy [5, 24]. In our study, we defined treatment success as freedom from signs or symptoms of infection after the end of treatment. Recently, treatment success has been proposed by an expert panel as the microbiological and clinical eradication of infection without relapsed infection, freedom from subsequent surgical intervention for the same infection, and freedom from mortality related to the PJI [24-26].

Next to infection control, which is certainly the primary aim of all treatment protocols, additional goals should be the restoration of function and minimizing infection-related morbidity and mortality $[27,28]$. Unfortunately, these objectives are not possible for all patients suffering from PJI. Surgical treatment options, although often required, may not be possible or appropriate due to severe comorbidities and a resulting increased perioperative risk. These patients may be best managed conservatively, either by long-term antibiotic suppression, acceptance, or even the creation of a new chronically discharging sinus $[5,24]$.

Although the technique of two-stage revision surgery seems to be well established for chronic PJI of the knee, different protocols exist among institutions with regard to debridement, spacer choice, and timing of reimplantation, as well as the definition or diagnostic tools used to indicate infection eradication. Failure rates of the two-stage protocol are between 10 and $40 \%$ according to recent studies [25, 29-31]. The factors associated with failure to ultimately achieve a successful second-stage revision after initial arthroplasty resection are not well understood [31]. One suggested reason for treatment failure is a persisting infection, often with the same causative organism(s) [3, 32-35]. Another reason for persisting infection following two-stage treatment could be the high incidence of false-negative results of pre- and intraoperative tissue samples with regard to the infection-causing microorganisms. If the biopsy misses the small biofilm population among the involved tissue, there will be a false-negative culture [36]. Foregoing antimicrobial therapy or errors during sampling and processing of the specimens may also lead to false results [37-40]. Sonication represents a huge advantage in terms of identification of infection-causing microorganisms.

Unfortunately, joint spacers, as they are used to bridge the time between implant removal and 
replantation, may also develop biofilms [26, 41, 42]. Therefore, the timing of second-stage surgery, and thus the spacer retention period, seems to be of high relevance for treatment success. Furthermore, in cases of PJI, clinicians must remember that standard antimicrobial-susceptibility tests cannot be used to reliably predict the outcome. Ideally, the antimicrobial agent should have bactericidal activity against surface-adhering, slow-growing, and biofilm-producing microorganisms. The presence of difficult-to-treat germs as well as undetected infection-causing microorganisms may therefore negatively influence the effect of the antimicrobial treatment [5, 43-46].

In our institutional review, we identified the following as risk factors for failure to achieve an infection-free two-stage revision: a prolonged spacer-retention, McPherson extremity grade 3, and unidentified/difficult-to-treat microorganisms. We observed the best outcome regarding infection-control in patients who had undergone second-stage surgery within 12 weeks of the first-stage surgery. Nearly $90 \%$ of these patients stayed free from infection until the final follow-up. Although most authors have suggested spacer-retention periods of 6 weeks, many of our patients exceeded this recommended period of time before completing the two-stage protocol [3-5, 13]. This circumstance may be explained by several factors: a bad general condition, which coincides with an increased perioperative risk, persisting infection, and not regularly appearing for follow-up examination within first- and second stage surgery. Furthermore, an increased number of performed spacer-exchanges, as well as a bad local extremity grade, exerted a significant negative impact on the outcome as well in the investigated cohort.

We observed an overall reinfection rate of almost $20 \%$ following the two-stage exchange protocol for PJI of the knee. This finding is in line with current literature $[3,9,13,14,23,25,31]$. However, due to the retrospective study-design and the variable individual factors which may have also affected the outcome, these findings have to be considered with care. Further prospective studies would be desirable to support our findings.

\section{Conclusion}

We observed the best outcome regarding infection control in patients who had undergone second-stage surgery within 12 weeks after first-stage surgery. Nearly $90 \%$ of these patients stayed free from infection until final follow-up. An increased number of performed spacer-exchanges and a bad local extremity grade seemed to have a negative impact on the outcome regarding infection control.

\section{Acknowledgements}

\section{Author Contributions}

IV performed the data recruitment and wrote the main manuscript. GS performed all statistical analyses. MG contributed to the manuscript with his great knowledge of prosthetic infections. PS, UB, and AL critically revised the manuscript.

\section{Ethical approval}

Ethical approval was obtained from the Ethics Committee of the Medical University of Graz.

\section{Availability of data and material}

The datasets generated during and/or analysed during the current study are available from the corresponding author on reasonable request.

\section{Competing Interests}

The authors have declared that no competing interest exists.

\section{References}

1. Parvizi J. Periprosthetic joint infection of the hip and knee. New York: Springer; 2014.

2. Kurtz SM, Lau E, Schmier J, et al. Infection burden for hip and knee arthroplasty in the United States. J Arthroplasty. 2008;23(7):984-991.

3. Warth LC, et al. Two-stage treatment for total knee arthroplasty infection utilizing an articulating prefabricated antibiotic spacer. J Arthroplasty. 2020;35(3):57-62

4. Trampuz A, Zimmerli W. Diagnosis and treatment of implant-associated septic arthritis and osteomyelitis. Curr Infect Dis Rep. 2008:10:394-403.

5. Zimmerli W, Trampuz A, Ochsner PE. Prosthetic-Joint Infections. N Engl J Med. 2004;351:1645-1654

6. Cobo J, Miguel LG, Euba G, et al. Early prosthetic joint infection: outcomes with debridement and implant retention followed by antibiotic therapy. Clin Microbiol Infect. 2011;17:1632-1637.

7. Hsieh $\mathrm{PH}$, Lee MS, Hsu KY, et al. Gram-negative prosthetic joint infections: risk factors and outcome of treatment. Clin Infect Dis. 2009·49:1036-1043.

8. Westrich GH, Salvati EA, Brause B. Postoperative infection. In: Bono JV, McCarty JC, Thornhill TS, et al., editors. Revision total hip arthroplasty. New York: Springer-Verlag; 2008. p. 371-390.

9. Aalirezaie A, Abolghasemian M, Busato, et al. Hip and knee section, treatment, two-stage exchange: proceedings of inter- national consensus on orthopedic infections. J Arthroplasty. 2019;34:439-443.

10. McPherson EJ, Tontz W Jr, Patzakis M, et al. Outcome of infected total knee utilizing a staging system for prosthetic joint infection. Am J Orthop. 2008;28(3):161-165.

11. Parvizi J, Zmistowski B, Berbari EF, e al. New definition for periprosthetic joint infection-from the Workgroup of the Musculoskeletal Infection Society. Clin Orthop Relat Res. 2011;469:2992-2994.

12. Elgeidi A, Elganainy AE, Abou Elkhier N, Rakha S. Interleukin-6 and other inflammatory markers in diagnosis of periprosthetic joint infection. Int Orthop. 2014;38(12):2591-2595.

13. Kildow BJ, Della-Valle CJ, Springer BD. Single vs 2-stage revision for the treatment of periprosthetic joint infection. J Arthroplasty. 2020:35(3S) 24-30.

14. Romano CL, Gala L, Logoluso N, Romano D, Drago L. Two-stage revision of septic knee prosthesis with articulating knee spacers yields better infection eradication rate than one-stage or two-stage revision with static spacers. Knee Surg Sports Traumatol Arthrosc. 2012;20:2445-2453.

15. Kusuma SK, Ward J, Jacofsky M, Sporer SM, Della Valle CJ. What is the role of serological testing between stages of two-stage reconstruction of the infected prosthetic knee? Clin Orthop Relat Res. 2011·469:1002e.

16. Lausen B, Schumacher M. Maximally selected rank statistics. Biometrics. 1982;48:73-85.

17. Hothorn T. axstat: Maximally selected rank statistics. $\mathrm{R}$ package version 0.7-18 2013; http://cran.rproject.org/web/packages/maxstat/.

18. Joseph TN, Chen AL, Di Cesare PE. Use of antibiotic-impregnated cement in total joint arthroplasty. J Am Acad Orthop Surg. 2003;11:38-47.

19. Anagnostakos K, Fink B. Antibiotic-loaded cement spacersdlessons learned from the past 20 years. Expert Rev Med Devices. 2018;15:23-245.

20. Hofmann AA. Two-stage exchange is better than direct exchange in the infected THA. Orthopedics. 1999:22:918. 
21. Moyad TF, Thornhill T, Estok D. Evaluation and management of the infected total hip and knee. Orthopedics. 2008;31:581-588.

22. Klouche S, Sariali E, Mamoudy P. Total hip arthroplasty revision due to infection: a cost analysis approach. Orthop Traumatol Surg Res. 2010;96:124-132.

23. Fowler TJ, Sayers A, Whitehouse MR. Two-stage revision surgery for periprosthetic joint infection following total hip arthroplasty. Ann Transl Med. 2019;7(8):S261.

24. Xu C, Goswami K, Li WT, et al. Is treatment of periprosthetic joint infection improving over time? J Arthroplasty. 2020. doi:10.1016/j.arth.2020.01.080.

25. Wang Q, Goswami K, Kuo FC, Xu C, Tan TL, Parvizi J. Two-stage exchange arthroplasty for periprosthetic joint infection: the rate and reason for the attrition after the first stage. J Arthroplasty. 2019;34:2749-2756.

26. Ekpo TE, Berend KR, Morris MJ, Adams JB, Lombardi AV. Partial two-stage exchange for infected total hip arthroplasty: a preliminary report. Clin Orthop Relat Res. 2014;472:437-448.

27. Tande AJ, Pate IR. Prosthetic joint infection. Clin Microbiol Rev. 2014;27(2):302-345.

28. Gehrke T, Alijanipour P, Parvizi J. The management of an infected total knee arthroplasty. Bone Joint J. 2015;97B:20-29.

29. Cancienne JM, Werner BC, Bolarinwa SA, Browne JA. Removal of an infected total hip arthroplasty: risk factors for repeat debridement, long-term spacer retention, and mortality. J Arthroplasty. 2017;32:2519-2522.

30. Gomez MM, Tan TL, Manrique J, Deirmengian GK, Parvizi J. The fate of spacers in the treatment of periprosthetic joint infection. J Bone Joint Surg Am. 2015;97:1495e502.

31. Barton CB, Wang DL, An Q, Brown TS, Callaghan JJ, Otero JE. Two-stage exchange arthroplasty for periprosthetic joint infection following total hip or knee arthroplasty is associated with high attrition rate and mortality. J Arthroplasty. 2019. doi:10.1016/j.arth.2019.12.005.

32. Fink B, Grossmann A, Fuerst M, Schäfer P, Frommelt L. Two-stage cementless revision of infected hip endoprostheses. Clin Orthop Relat Res. 2009;467:1848-1858.

33. Moran E, Byren I, Atkins BL. The diagnosis and management of periprosthetic infections. J Antimicrob Chemother. 2007:65(3):45-54.

34. Masri BA, Panagiotopoulos KP, Greidanus NV, Garbuz DS, Duncan CP. Cementless two-stage exchange arthroplastyfor infection after total hip arthroplasty. J Arthroplasty. 2007;22:72-78.

35. Kraay MJ, Goldberg VM, Fitzgerald SJ, Salata MJ. Cementless two-staged total hip arthroplasty for deep periprostheticinfection. Clin Orthop Relat Res. 2005;441:243-249.

36. Huang $\mathrm{R}$, Hu CC, Adeli B, Mortazavi J, Parvizi J. Culturenegative periprosthetic joint infection does not preclude infection control. Clin Orthop Relat Res. 2012;470:2717-2723.

37. Parvizi J, Alijanipour P, Barberi EF. Novel developments in the prevention, diagnosis and tretment of periprosthetic joint infections. J Am Acad Orthop Surg. 2015;23:32-43.

38. Legout L, Sennevillem E. Periprosthetic joint infections: clinical and bench research. Sci World J. 2013;2013:549091.

39. Lass R, Giurea A, Kubista B. Bacterial adherence to different components of total hip prosthesis in patients with prosthetic joint infection. Int Orthop. 2013:38(8):1597-1602.

40. Maier GS, Horas K, Seeger JB, Roth KE, Kurth AA, Maus U. Is there an association between periprosthetic joint infection and low vitamin D levels? Int Orthop. 2014;38(7):1499-1504.

41. Nelson CL, Jones RB, Wingert NC, Foltzer M, Bowen TR. Sonication of antibiotic spacers predicts failure during two-stage revision for prosthetic knee and hip infections. Clin Orthop Relat Res. 2014;472:2208-2214.

42. Mariconda $\mathrm{M}$, Ascione $\mathrm{T}$, Balato $\mathrm{G}$, et al Sonication of antibiotic-loaded cement spacers in a two-stage revision protocol for infected joint arthroplasty. BMC Musculoskelet Disord. 2013;14(193):1471-1474.

43. Diaz-Ledezma C, Higuera CA, Parvizi J. Success after treatment of periprosthetic joint infection: A Delphi-based international multidisciplinary consensus. Clin Orthop Relat Res. 2013;471:2374-2382.

44. Costerton JW, Stewart PS, Greenberg EP. Bacterial biofilms: a common cause of persistent infections. Science. 1999;284:1318-1322.

45. Nelson CL, Jones RB, Wingert NC, Foltzer M, Bowen TR. Sonication of antibiotic spacers predicts failure during two-stage revision for prosthetic knee and hip infections. Clin Orthop Relat Res. 2014;472:2208-2214.

46. Kendall RW, Duncan CP, Beauchamp CP. Bacterial growth on antibioticloaded acrylic cement: a prospective in vivo retrieval study. J Arthroplasty. 1995;10:817-822. 